Ein zweiter Auswuchs ist die intravenöse Infusion von Mistelpräparaten für eine besonders schnelle und ausgeprägte Wirksamkeit. Es erschließt sich mir nicht, weshalb ein Präparat wie Viscum album, das nur für die subkutane Injektion zugelassen ist und dort keinen Wirksamkeitsnachweis erbracht hat, durch die nicht zugelassene intravenöse Gabe eine besonders starke Wirksamkeit entfalten soll.

- Dr. med. Rainer Hakimi, Facharzt für Allgemeinmedizin - Naturheilverfahren Psychotherapie - Ärztliches Qualitätsmanagement, Leitender Gesellschaftsarzt Hallesche Krankenversicherung a. G., Reinsburgstraße 10, D-70178 Stuttgart

\title{
Warum sollten Studien zur Misteltherapie nicht verblindbar sein?
}

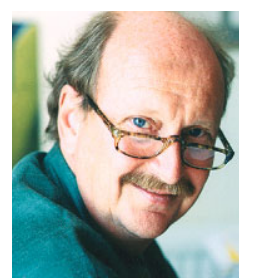

Prof. Dr. med. E. Ernst Peninsular Medical

School,

University of

Exeter/UK

- Wiebelitz und Beer meinen, dass eine „Misteltherapie stets empfehlenswert" sei, und vertreten folgende Auffassung: „Da die prozessstandardisierten Präparate individuell nach Wirkungen dosiert werden, ist eine Verblindung im Sinne von randomisierten kontrollierten Studien praktisch nicht erreichbar.“ Da Ähnliches von „Naturheilkundlern“ häufig behauptet wird, lohnt es sich, diesen Satz genauer zu analysieren.

Was bedeutet „eine Verblindung im Sinne von randomisierten kontrollierten Studien"? Verblindung und Randomisierung sind zwei verschiedene Sachen. Eine Studie mag verblindet, aber nicht randomisiert sein; eine andere könnte randomisiert, aber nicht verblindet durchgeführt werden.

Warum sollen prozessstandardisierte, individuell nach Wirkung dosierte Therapien nicht verblindbar sein? Der Sinn einer verblindeten Studie ist es, Klarheit darüber zu schaffen, ob das Verum der Therapie in der Kontrollgruppe (z.B. Placebo) überlegen ist. Selbst wenn individuell nach Wirkung dosiert wird, können Placeboeffekte dennoch gegen Verumeffekte abgewogen werden. Man appliziert beide Therapieformen in ansteigenden Dosierungen, bis der Patient Effekte angibt, die eine ausreichende Dosis anzeigen. Da Placeboeffekte zu erwarten sind, sollten in beiden Gruppen Wirkungen auftreten.

- Prof. Dr. med. Edzard Ernst, MD, PhD, FMedSci, FSB, FRCP, FRCPEd, Complementary Medicine, Peninsula Medical School, Universities of Exeter \& Plymouth, 25 Victoria Park Road, Exeter EX2 4NT 